\title{
Metabolic Vascular Syndrome: New Insights into a Multidimensional Network of Risk Factors and Diseases
}

\author{
Gerhard H. Scholz ${ }^{a, b} \quad$ Markolf Hanefeld ${ }^{c, d}$ \\ ${ }^{a}$ Department of Endocrinology, Diabetology, Cardiology and General Medicine, St. Elisabeth-Krankenhaus Leipzig, Leipzig, Germany;

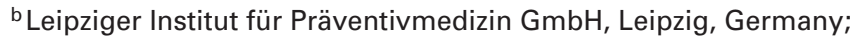 \\ ' GWT-TUD GmbH, Dresden Technical University, Dresden, Germany; \\ d Medical Clinic III, University Hospital Carl Gustav Carus, Dresden, Dresden, Germany
}

\section{Keywords}

Metabolic syndrome - Metabolic vascular syndrome .

Obesity · Insulin resistance · Type 2 diabetes · Common soil

\section{Summary}

Background: Since 1981, we have used the term metabolic syndrome to describe an association of a dysregulation in lipid metabolism (high triglycerides, low highdensity lipoprotein cholesterol, disturbed glucose homeostasis (enhanced fasting and/or prandial glucose), gout, and hypertension), with android obesity being based on a common soil (overnutrition, reduced physical activity, sociocultural factors, and genetic predisposition). We hypothesized that main traits of the syndrome occur early and are tightly connected with hyperinsulinemia/insulin resistance, procoagulation, and cardiovascular diseases. Methods: To establish a close link between the traits of the metabolic vascular syndrome, we focused our literature search on recent original work and comprehensive reviews dealing with the topics metabolic syndrome, visceral obesity, fatty liver, fat tissue inflammation, insulin resistance, atherogenic dyslipidemia, arterial hypertension, and type 2 diabetes mellitus. Results: Recent research supports the concept that the metabolic vascular syndrome is a multidimensional and interactive network of risk factors and diseases based on individual genetic susceptibility and epigenetic changes where metabolic dysregulation/metabolic inflexibility in different organs and vascular dysfunction are early interconnected. Conclusion: The metabolic vascular syndrome is not only a risk factor constellation but rather a life-long abnormality of a closely connected interactive cluster of developing diseases which escalate each other and should continuously attract the attention of every clinician.

(c) 2016 S. Karger GmbH, Freiburg

\section{Introduction}

After a long history of attempts to bring together different metabolic disorders such as diabetes, gout, glucose intolerance, and insulin resistance with central obesity and fatty liver as well as hypertension and cardiovascular diseases (CVD), one of the authors of this overview described this syndrome already in 1981 as follows: 'The metabolic syndrome represents the common prevalence of obesity, hyper- and dyslipoproteinemia, maturity-onset diabetes (type 2), gout, and hypertension associated with increased incidence of atherosclerotic vascular disease, fatty liver, and gallstones that develops on the basis of genetic susceptibility combined with overnutrition and physical inactivity. If this working hypothesis can be confirmed, it provides the basis for integrated diagnostics and prevention of this cluster of diseases which is of central importance for health care' (fig. 1) [1].

35 years later, after many evolutionary changes in definition and some critical discussions about the usefulness of the term metabolic syndrome and its interpretation in clinical practice, we would like to conclude that the initial and primary idea of a tight and early connection between metabolic dysregulation and vascular dysfunction has now a more convincing and broader scientific basis and has found its way into the clinical evaluation of patients. If nothing else, it can be translated as a guide to individualized riskadjusted treatment of these diseases.

In the Diabetes in Germany (DIG) study, eleven different combinations of the main traits of the metabolic syndrome were identified in 4,020 unselected patients aged 35-80 years with type 2 diabetes [2]. Based on the American Heart Association/National Heart, Lung, and Blood Institute definition [3], the prevalence of the overall metabolic syndrome in this group was $74.4 \%$, with an odds ratio for atherosclerotic vascular diseases of 1.41 and a wide range of odds ratios for other clusters (0.47-10.9).

With better knowledge on the molecular mechanism how the different clusters are related and how a probably early interaction

\section{KARGER}

(๑) 2016 S. Karger GmbH, Freiburg
Prof. Dr. med. Gerhard H. Scholz Abteilung Innere Medizin I

St. Elisabeth-Krankenhaus Leipzig

Biedermannstraße 84, 04277 Leipzig, Germany gerhard.scholz@onlinehome.de 


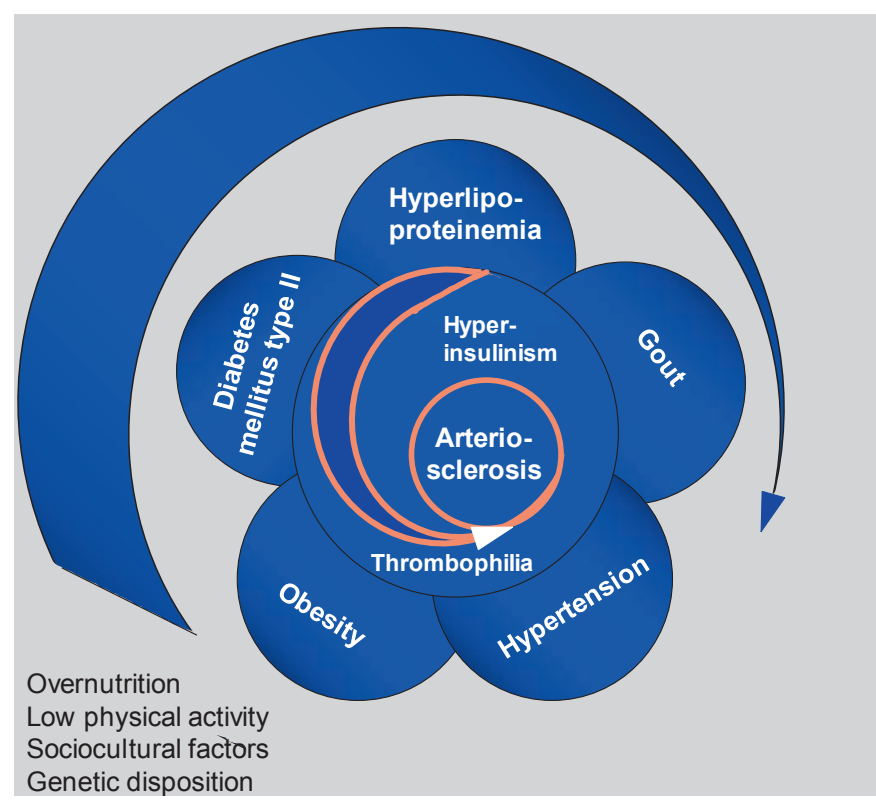

Fig. 1. Historic description of the metabolic syndrome (adapted from [1]).

takes place between disturbed metabolism and dysfunctional vasculature, we believe that much more attention should be directed to the processes leading to this interplay. This holds true also for the treatment of the different traits of the syndrome expressed as different combinations of central obesity, atherogenic dyslipidemia, hypertension, and hyperglycemia regarding positive and negative pharmacological interactions of selected medications. These were the reasons why in 2007, a group of clinicians and scientists in Saxony (Germany) established the term metabolic vascular syndrome, developed and published a special practice guideline [4], integrated the concept into special antidiabetic treatment strategies [5], and used it to guide an individualized treatment [6]. In our definition of the metabolic vascular syndrome, we traditionally included type 2 diabetes as part of this syndrome since we are convinced that also in most of the type 2 diabetic patients the abovementioned typical metabolic dysfunctions are causative and lasting (fig. 2).

Because our definition also includes impaired fasting glucose and impaired glucose tolerance as part of the syndrome, for these prediabetic patients the individual syndrome traits can be used to calculate the diabetes risk, which is considerably higher if glucose homeostasis is dysregulated [7].

\section{Traits, Genetic Susceptibility, and Insulin Resistance}

We do not deny the importance and practical value of defining critical values for the main traits of the metabolic syndrome (waist circumference adapted for ethnicity, blood pressure, serum concentrations of triglycerides and high-density lipoprotein (HDL) cholesterol as well as plasma glucose values) for a worldwide unifying 'definition' of the metabolic syndrome and early prevention [8]. However, we believe that the understanding of how the different traits interact and how this syndrome can be treated is more important than counting the number of components (e.g. 3 out of 5) and making a diagnosis. Without any doubt the metabolic syndrome is more than a cluster of premorbid risk factors with type 2 diabetes as a major cardiovascular risk factor as a key component.

Coming back to our roots in 1981, our understanding of the concept of the metabolic (vascular) syndrome includes the genetic predisposition or susceptibility to the different traits as well as insulin resistance (hyperinsulinemia as a consequence) as part of the pathophysiological background. Insofar, our earlier description and our current concept of the metabolic vascular syndrome include many of the ideas presented by the pioneers of the insulin resistance syndrome $[9,10]$.

\section{Selective Insulin Resistance in Liver, Muscle, and Adipose Tissue as a Cornerstone of the Metabolic Vascular Syndrome}

Insulin resistance is defined as a clinical state in which a normal or elevated insulin level produces an attenuated biological response [11]. Because insulin-stimulated glucose uptake can easily be measured, many of the studies on insulin resistance were related to this insulin action. However, insulin stimulates or inhibits a much greater variety of metabolic pathways in many tissues. Metabolic pathway-selective insulin resistance is now becoming more and more important in the understanding of the complex network of the metabolic vascular syndrome.

In the liver, insulin normally stimulates glucose uptake, glucose oxidation, and glycogen synthesis, suppresses gluconeogenesis, and stimulates triglyceride synthesis and secretion via very-low-density lipoproteins (VLDL).

Fatty acid-induced insulin resistance in the liver and muscle is mainly related to enhanced fatty acid uptake and production of diacylglycerol (DAG), which interacts with further intracellular signaling (for review see [12]). In short, in the liver, DAG stimulates protein kinase $\mathrm{C} \varepsilon(\mathrm{PKC} \varepsilon)$, which inhibits the activation of insulin receptor substrates (IRS 1 and 2) and pAkt2, leading to the suppression of glycogen synthesis and reduced glycogen storage but also to a diminished suppression of the Forkhead box protein $\mathrm{O} 1$ (FOXO1) and a reduction in the suppression of gluconeogenesis. The net effect of insulin resistance is an enhanced glucose output from the liver. In contrast, triglyceride synthesis and VLDL release remain intact and are even enhanced. This metabolic paradox is called selective insulin resistance and was explained by the upregulation of SREB-1c [13], with the suggestion of mTORC1 kinase being the branch point [14].

Another explanation of the 'selective insulin resistance' paradox in the liver was recently suggested [15]. In a model of mice with completely deleted insulin signaling intermediates Akt and/or FOXO1, mice lacking Akt in the liver were hyperglycemic after carbohydrate feeding, an effect that was lost by FOXO1 deletion. Mice lacking only Akt had an 80\% reduction in de novo lipogenesis 


\section{The metabolic vascular syndrome (MVS)}

Fig. 2. The multidimensional network of the metabolic vascular syndrome.

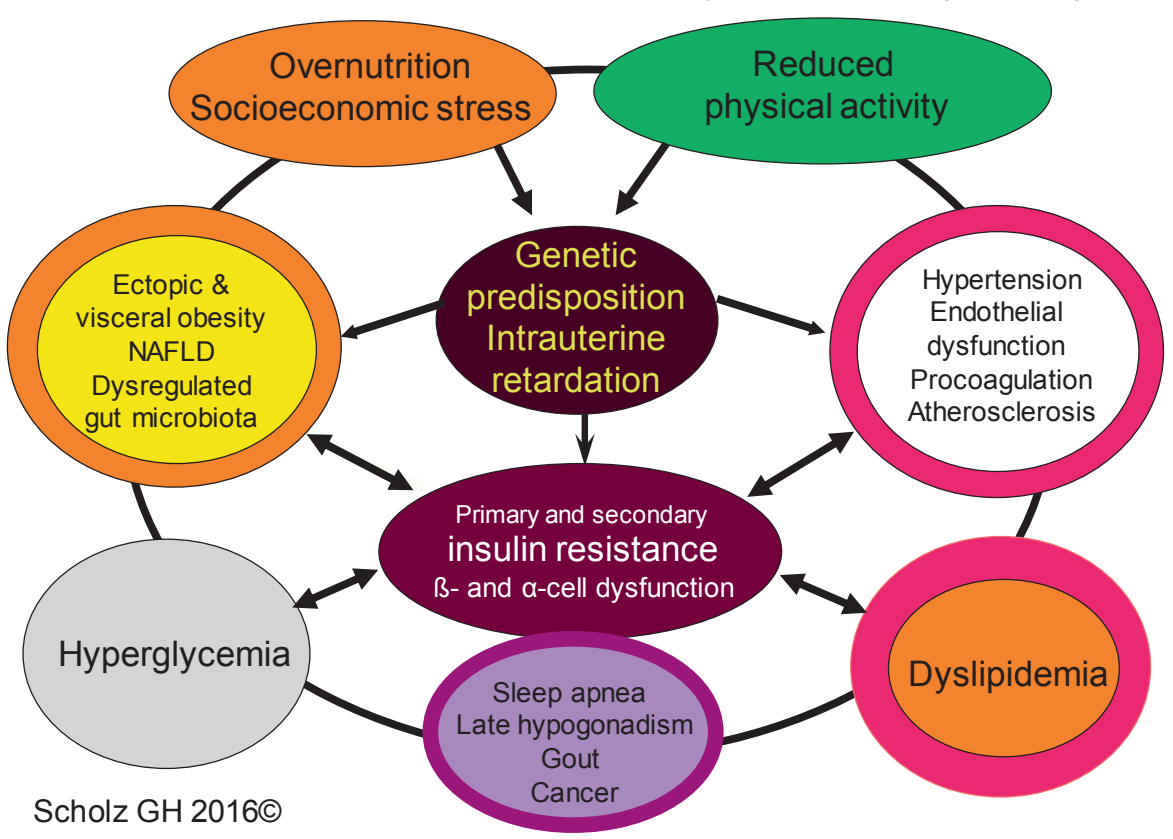

but additional FOXO1 deletion had no effect on reduced lipogenesis. From these and other results the authors concluded that liver insulin signaling through Akt is essential for hepatic lipid synthesis even in the absence of FOXO1 or if FOXO1 is inhibited. Interestingly, insulin was able to suppress hepatic glucose production even in insulin signaling-deficient mice, so the concept of a cell-nonautonomous control of hepatic glucose production under conditions of insulin resistance evolved. In further studies it could be shown that insulin suppression of hepatic glucose production in vivo was independent of vagal stimulation and glucagon in livers deficient in insulin action but dependent on the regulation of circulating fatty acids coming from other tissues by insulin.

In the muscle, insulin resistance is induced by high fatty acid uptake, DAG-activated PKC $\theta$, and the following suppression of pIRS1, lower Akt2, reduced GLU4 activation, and reduced activity of glycogen synthase with the net effect of reduced glucose uptake and glycogen storage as well as enhanced triglyceride accumulation. Skeletal muscle insulin resistance is strongly related to hepatic de novo lipogenesis, increasing liver triglyceride synthesis, and reduced HDL concentrations (for recent review see [12]).

Selective insulin resistance exists in adipocytes. In a special experimental setting, all models of insulin resistance in adipocytes showed a reduced glucose uptake at the maximum dose of insulin. On the contrary, other major actions of insulin were normal, e.g. protein synthesis and antilipolysis, although with an already known higher sensitivity for stimulation (protein synthesis) and inhibition (antilipolysis) compared to the stimulation of glucose uptake [16]. The authors concluded that the primary defect in this kind of selective insulin resistance should be on the level of glucose transporter activation and/or cycling. Recent observations that the intracellular sorting receptor SORLA is involved in the insulin re- ceptor (IR) translocation from endosomes to the plasma membrane and can modulate insulin-dependent rates of lipolysis in white adipose tissue opens up a new pathway of selective insulin resistance [17]. Another candidate involved in the development of adipocyte insulin resistance is IRF3, a transcriptional regulator of immune response genes which can drive the inflammatory response within the adipocyte [18]. By means of binding to toll-like receptors (TLR), saturated fatty acids can induce endoplasmic stress by stimulating intracellular signaling cascades (e.g. c-Jun $\mathrm{N}$ terminal kinase (JNK), IкB kinase $\beta$ (IKK $\beta$ ) and are obviously able to induce adipocyte insulin resistance and inflammatory responses [19]. Last but not least, DNA damage (telomere shortening and/or DNA double-strand breaks), which is already visible at the onset of obesity, and activation of the p53 pathway might contribute to insulin resistance in adipocytes with reduced glucose uptake, increased lipolysis, enhanced release of glycerol and free fatty acids (FFA), and progression of inflammation [20].

\section{Selective Vascular Insulin Resistance}

Another important factor, i.e. selective vascular insulin resistance, was found to be a key mediator for the development of vascular dysfunctions in diabetes [21]. Because insulin resistance is early evolving in some traits of the metabolic vascular syndrome and hyperinsulinemia is often observed in obesity, most of the molecular mechanisms discussed in this comprehensive review are present in the metabolic vascular syndrome and should lead to the same results.

Normally, endothelial and vascular smooth muscle and pericyte functions are in a balanced state. Through activation of the IR, two cascades of signaling molecules are activated. 


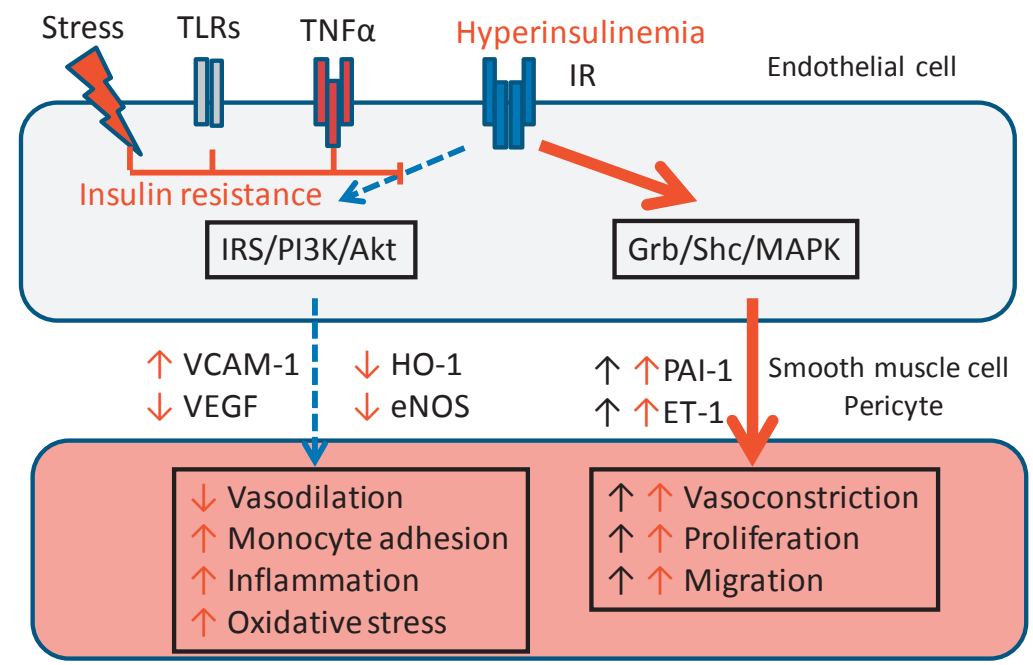

Fig. 3. Selective vascular insulin resistance (adapted from [21]).

\section{Antiatheroscleroticaction Proatherosclerotic action}

\section{Dysbalanced situation with metabolic and vascular inflexibility}

One cascade involving IRS, phosphatidylinositol-3-kinase (PI3K), and protein kinase B (Akt) leads to suppression of vascular cell adhesion molecule 1 (VCAM-1) and stimulation of vascular endothelial growth factor (VEGF), modulates heme oxygenase 1 (HO-1), and stimulates expression of endothelial nitric oxide synthase (eNOS). This first cascade is responsible for vasodilation and reduction of monocyte adhesion, inflammation, and oxidative stress of vascular smooth muscles and protects against atherosclerotic changes of the vessel wall (antiatherosclerotic action).

In contrast, the second cascade, consisting of growth factor receptor-bound protein 2 (GRB2), Shc (a molecular adaptor protein), and mitogen-activated protein kinase (MAPK), enhances plasminogen activator inhibitor and endothelin-1, leading to vasoconstriction, cell proliferation, and migration, and has proatherosclerotic potency.

Both cascades allow a flexible adaptation of blood vessels during the dynamic changes in blood flow and energy supply but also during necessary developmental and repair processes.

Insulin resistance, induced in endothelial cells by stress, pathogenic activation of TLR, or inflammatory stimulation of tumor necrosis factor- $\alpha$ (TNF- $\alpha$ ) receptors, inhibits the first cascade and reduces the protective antiatherosclerotic action. As the second cascade is not influenced by insulin resistance, its proatherosclerotic effects remain active. The inhibition or even inversion of the effects of the first cascade lead to a reduced metabolic flexibility of the endothelial cell to normal stimuli and to an imbalance in the regulation of smooth muscle activity towards atherosclerosis. The balance is even more disturbed if more severe hyperglycemia (hyperglycemic stress) enhances endothelial insulin resistance (glucotoxic form) and suppresses the first cascade. Additionally, hyperinsulinemia (as in obesity and in systemic insulin resistance) can stimulate the second cascade, leading to an exaggerated proatherosclerotic process (fig. 3). This model is able to explain why many of the patients already have CVD in the early non-diabetic stage of the metabolic vas- cular syndrome. It also explains why even moderate hyperglycemia (e.g. impaired fasting glucose and/or impaired glucose tolerance) heightens the risk of more CVD, as recently stated in a very profound pro and contra discussion on the term prediabetes [22, 23].

The development of diabetes from a prediabetic stage adds the special problems of a marked diminished availability of insulin and profound hyperglycemia to the above-mentioned cardiovascular risks of an 'early' metabolic vascular syndrome.

There are many similarities in the pathogenetic mechanisms of selective vascular insulin resistance and severe insulin deficiency, which can easily be derived from the model explained above. A reduced or missing activation of the IRS/PI3K/Akt cascade in insulin signaling, whether by insulin resistance or by insulin deficiency, will ultimately promote the proatherogenic processes [21].

Vascular pathology with insulin resistance also exists as primary part of a common soil of the metabolic vascular syndrome and is not simply a consequence of insulin resistance syndrome, dyslipidemia, or hypertension as atherogenic traits.

\section{Obesity and the Metabolic Vascular Interplay}

One of the main traits of the metabolic vascular syndrome is obesity. The adipocyte is now accepted as a 'professional' secretory cell (for review see [24]) with broad adaptive capacity for fuel storage and release (white adipocytes) and energy/temperature regulation (brown and beige adipocytes). In contrast, adipose tissue, composed of adipocytes, fibroblasts as well as endothelial, angiogenic, and immune cells, is also very actively involved in the whole body regulation of metabolism and immunity. Whereas a normal body fat composition is healthy and even helpful in situations of starving and infections, diminished or dystrophic fat, e.g. in the case of lipodystrophy, is harmful. On the contrary, overnutrition and reduction of 


\section{Visceral fat accumulation as indicator and driving force of the metabolic vascular syndrome}

Fig. 4. Effects of visceral obesity and ectopic fat depots on liver, vasculature, and muscle. RAS = Renin-angiotensin system; SNS = sympathetic nervous system; VAT $=$ visceral adipose tissue; FFA = free fatty acids; IL6 = interleukin 6; $\mathrm{CRP}=\mathrm{C}$-reactive protein; ATII = angiotensin II; PA-I = plasminogen activator inhibitor.

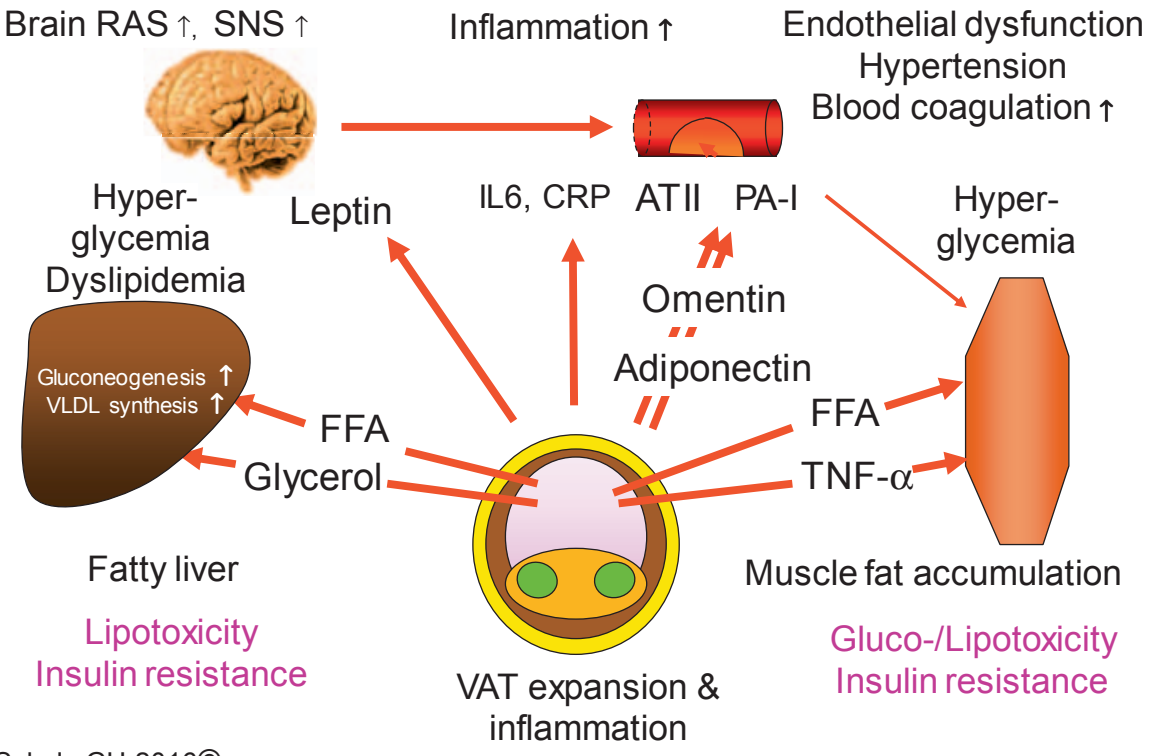

Scholz GH 2016(C) physical activity in connection with socioeconomic stress have led to a worldwide obesity epidemic and, on the individual level, to an increasing adaptation failure of the fat tissue with deleterious health consequences. Obviously, obesity is a common risk factor for CVD and cancer. However, not all obese patients seem to have an increased risk of CVD and cancer. Therefore, it seemed reasonable to differentiate between 'healthy obese' (HOB) and 'unhealthy obese' (UHOB) individuals $[25,26]$. This concept stimulated an intensive research for protective and pathogenic factors during the development of obesity (for review see [27]). Recent results from longitudinal studies and meta-analyses regarding the long-term outcome in HOB individuals suggested an even more complex situation, because a considerable part of the initially $\mathrm{HOB}$ patients switched over time (e.g. during 10 years) to the UHOB state whereas others show a higher degree of protection $[28,29]$. That HOB might be a transient state at least for some HOB patients is supported by the observation of an only quantitative difference in obese patients with and without insulin resistance in insulin-stimulated gene expression but a considerable qualitative difference to normal-weight individuals [30]. This suggests an overall pathogenetic role for a more than normal fat accumulation. Critical expansion of visceral adipose tissue (VAT), leading to the visceral adiposity syndrome (for review see [31]), seems to play a major role in the development of the metabolic vascular syndrome. Compared with subcutaneous adipose tissue (SAT), VAT is characterized by higher catecholamine-induced lipolytic activity and diminished antilipolytic effects of insulin, making VAT very sensitive to hormonal and neural changes during fasting and feeding. Additionally, whereas SAT is more efficient in the uptake of FFA, VAT has a higher capacity for glucose uptake and FFA release. In overfeeding, if the energy storage capacity of subcutaneous tissue is exhausted, VAT becomes the prominent energy store and the main cause of lipotoxic insulin resistance in the liver and other organs. Thus, VAT is an indicator and a driving force for the metabolic vascular syndrome (fig. 4).

In addition to the visceral fat compartment (surrounding the gut), there is also a massive increase in ectopic fat deposition in the liver, pancreas, epicardium, and pericardium as well as in the renal sinus. All these depots can contribute to local and systemic insulin resistance and organ pathology (recently reviewed in [32]).

According to the first description of the term metabolic syndrome [1], abnormal hepatic fat accumulation is traditionally accepted as an important part of the syndrome. At least three mechanisms are involved in normal fat metabolism, ectopic fat accumulation, and selective insulin resistance in the liver, as recently pointed out in the following model [12]. Via direct insulin signaling, de novo lipogenesis in hepatocytes generates triglycerides from excess carbohydrate substrates and promotes VLDL synthesis and secretion to adipose tissue. This is the direct insulin-dependent pathway. Via action in adipose and muscle tissue, however, insulin is indirectly involved in hepatic fat metabolism. To explain these indirect effects of insulin, other pathways must be taken into account how triglyceride synthesis in the liver works without insulin.

In these pathways, esterification of preformed (external) FFA is largely due to enhanced lipolysis, especially when there is overt delivery of FFA and glycerol in insulin-resistant adipose tissue. A second phenomenon is the only partly insulin-dependent regulation of hepatic lipogenesis by glucose and especially fructose through sterol regulatory element-binding protein-1c (SRBP-1c) expression. This also explains why high fructose consumption is a part of the problem.

This diversification into direct and indirect insulin-dependent pathways can also explain why in the situation of hepatic insulin 
resistance, with failing suppression of glucose production by insulin, there is even an increase in liver fat accumulation. The increased turnover of fatty acids in the liver and the related enhancement of the lipid metabolite DAG seem to be responsible for selective insulin resistance in this organ. DAG can activate PKCe, the primary PKC isoform in the liver, and this PKC can bind and inhibit insulin receptor kinase activity and interrupt the normal insulin signaling pathway. Inhibition of this PKC can prevent high fatinduced non-alcoholic fatty liver disease (NAFLD) (for review see [33]).

NAFLD, especially if progressing to non-alcoholic steatohepatitis (NASH), is associated with increased concentrations of inflammatory cytokines (e.g. C-reactive protein (CRP), fibrinogen, monocyte chemoattractant protein 1 (MCP-1), TNF- $\alpha$ as well as betatrophin, fibroblast growth factor (FGF)-21, and fetuin A).

In summary, fatty liver is mainly the result of enhanced insulinindependent lipid pathways but is linked to systemic insulin resistance by enhanced supply of FFA from insulin-resistant visceral fat depots, reduced uptake of glucose in muscle as well as meal-related fructose load.

\section{Low-Grade Inflammation of Adipose Tissue: A Major Link to Vascular Dysregulation}

The following question arises: What is the link between dysregulated fat tissue expansion and organ dysfunction? Low-grade inflammation stimulated by dysfunctional adipose tissue was one of the most accepted working hypotheses to explain the connection between (central) obesity and vascular dysfunction [34]. 10 years later, in a scientifically profound recent review, it was convincingly worked out that there are reasonable arguments to conclude that adipose tissue inflammation following adipocyte hypertrophy after long-term positive energy balance is a main driving force for insulin resistance in fat tissue itself but also for systemic insulin resistance and probably also for an obesogenic memory [35]. Key factors which determine monocyte infiltration and the development of an inflammatorily active macrophage phenotype are positive energy balance, lipid accumulation in adipocytes, adipocyte hypertrophy, reduced insulin sensitivity of hypertrophic adipocytes, and processes related to hypoxia, autophagia, and apoptosis. One of the causes of this pathogenic process is the limited expendability of fat tissue. The adipocyte proliferative capacity is obviously genetically determined and limited. The only way to store excessive energy is via adipocyte hypertrophy. At some point in this development, when the inflammatory process is initiated and a typical pattern of activated macrophages evolves in adipose tissue, inflammation becomes a self-perpetuating cycle [36].

Bearing this in mind fat, tissue inflammation seems to be one of the leading causes of the metabolic-vascular syndrome, since many different secretory products of fat cells, such as proinflammatory cytokines, endothelial adhesion molecules, proatherogenic, procoagulative, and chemotactic mediators, and others, play a role in vascular dysfunction [37].
Inflammatory Cytokines, Adipokines, and the 'Adiponectin Paradox'

Observational studies in humans have shown a link between obesity, inflammatory markers, and CVD risk. CRP, which decreased the BMI-attributable cardiovascular risk, was predictive in an 11.4-year longitudinal population-based study, with a composite outcome comprising of the first event of fatal and non-fatal coronary heart disease as well as fatal and non-fatal stroke as the defined end points. However, there was no association with adiponectin or leptin concentrations [38]. Baseline TNF- $\alpha$, change from baseline TNF- $\alpha$ and baseline CRP, and elevated baseline adiponectin seem to be harmful and related to cardiovascular death, myocardial infarction, stroke, and coronary artery disease in patients with type 2 diabetes, whereas leptin and increase of leptin was protective over time. These effects were dependent on BMI for change from baseline of MCP-1, TNF- $\alpha$, and interleukin-6 (IL6) [39]. In a systematic review and meta-analysis of prospective studies to evaluate the overall quantitative contribution of adiponectin levels to the mortality risk in patients with CVD, increased baseline plasma adiponectin levels were significantly associated with an elevated risk of all-cause and cardiovascular mortality [40]. Other new data from patients with type 2 diabetes showed that the A allele of SNP rs822354 (in the ADIPOQ locus, which encodes for adiponectin) is associated with high serum adiponectin levels (both total and HMW isoform) and also with a high cardiovascular mortality rate after a follow-up period of 5.4 years [41].

Three 'paradox' situations for adiponectin evolved over the years [42]. One is the reduced adiponectin concentration with growing visceral fat mass. The second is the above-mentioned repetitive observation of an association of higher adiponectin levels with CVD, taking into account the antiatherosclerotic, anti-inflammatory, and antidiabetogenic properties of this adipokine. The third paradox is the strong association of adiponectin-inducing alleles of the $\mathrm{CDH} 13$ gene locus with traits that lead to metabolic (vascular) syndrome, which is an area of further research.

Omentin, another adipokine, is mainly expressed in stromal vascular cells in visceral fat depots [43]. In general, omentin is thought to have a protective function in CVD $[44,45]$. Omentin is also evident in human epicardial adipose tissue, with a reduced expression being related to coronary artery stenosis [46]. These observations contradict the results of a recent 3.5-year prospective observational study which showed that high plasma omentin concentrations might serve as a biomarker for an enhanced risk of cardiovascular mortality in patients with established or suspected stable coronary artery disease [47]. Leptin, about 20 years after its discovery as the key marker of the amount of energy stores with serum concentrations proportional to body fat mass but also regulated by short- and long-term food deprivation [48, 49], is known as an appetite-suppressing hormone with many other regulatory roles and fortunate effects in leptin-deficient humans (for short review see [50]).

Recent observations in humans and rodents have argued for a role in the development of hypertension as far as leptin was not only 
able to enhance the sympathetic nerve activity but also to upregulate the brain renin-angiotensin system during high-fat diet [51].

\section{Dysglycemia, Dyslipidemia, and Hypertension}

Considering the central role of visceral and ectopic fat accumulation and their contribution to the development of pathway-specific insulin resistance, it becomes clear that many of the features of the other traits of the metabolic vascular syndrome can be explained as long-term consequences of this kind of metabolic dysregulation. Recent epidemiological data support this concept of a timed sequence of the appearance of the main traits of the metabolic vascular syndrome [52]. In women, abdominal obesity and hypo- $\alpha$ lipoproteinemia were already observed in young adulthood, hypertension and hypertriglyceridemia in middle age, and diabetes later on. In men, abdominal obesity, hypo-a-lipoproteinemia, and hypertriglyceridemia occur in young adulthood, hypertension in middle age, and diabetes later. In a recent review, eleven factors are shown to be involved in the development of type 2 diabetes [53] Beta-cell failure with reduced insulin secretion, 'paradoxical' enhanced glucagon secretion, disturbed incretin function, changes in the metabolism of adipose tissue, muscle, liver, and brain, adaptation failure of the microbiome in the gut, immune dysregulation and inflammation, dysfunctions in the stomach and small intestine, and enhanced glucose reabsorption in the kidney are discussed. Many of these organs and tissues are directly or indirectly compromised by the systemic actions of the above-mentioned fat tissue dysfunction and related inflammation and of course by an unhealthy lifestyle as a main trigger of this adaptation failure.

The same is true for enhanced release of VLDL and pathological changes in VLDL catabolism as well as HDL and LDL metabolism related to insulin resistance. These are the causes of the 'atherogenic dyslipidemia complex' in the metabolic vascular syndrome, which includes the 'lipid triad' hypertriglyceridemia, low HDL cholesterol, and qualitative changes in LDL particles (small, dense LDL) as well as an accumulation of remnant lipoproteins and postprandial hyperlipidemia [54], accompanied by elevated apolipoprotein B and non-HDL cholesterol.
Although hypertension is a main trait of the metabolic vascular syndrome and has an important influence on the cardiovascular outcome, the relationship between the early traits and 'primary' hypertension is less clear [55]. Local renin-angiotensin systems are widely distributed, and one is also present in adipocytes (for review see [56]). Additionally, substrates from isolated adipocytes may directly stimulate the secretion of aldosterone $[57,58]$. The question of how these local systems interact with systemic RAAS is not fully understood. According to a recent investigation of systemic RAAS in obese humans, there was no association between visceral or subcutaneous fat tissue distribution and RAAS activity measures [59]. This does not exclude a harmful role of local or systemic RAAS activation during the development of the metabolic vascular syndrome because therapeutic RAAS interventions in these patients have shown protective cardiovascular effects. It is conceivable that selective vascular insulin resistance (fig. 3) can contribute to systemic hypertension in many different ways.

\section{Conclusion}

The metabolic vascular syndrome is more than a risk factor constellation. In our view, and based on our earlier concept of the metabolic syndrome from 1981, it is a lifelong existing cluster of risk factors and diseases caused by overnutrition, lower physical activity, and socioeconomic stress. The main traits, i.e. visceral and ectopic fat accumulation with low-grade inflammation, NAFLD, arterial hypertension, dysglycemia, and dyslipidemia, are related to genetic susceptibility and systemic and/or selective insulin resistance as well as a parallel development of changes in the vascular system even in early stages of the syndrome. A better understanding of the multidimensional network of the metabolic vascular syndrome will lead to better tailored and better integrated preventive and therapeutic strategies for multimorbid patients.

\section{Disclosure Statement}

The authors have nothing to disclose.

\section{References}

1 Hanefeld M, Leonhardt W: Das metabolische Syndrom. Dt Gesundheitswesen 1981;36:545-551.

2 Hanefeld M, Koehler C, Gallo S, Benke I, Ott P: Impact of the individual components of the metabolic syndrome and their different combinations on the prevalence of atherosclerotic vascular disease in type 2 diabetes: the Diabetes in Germany (DIG) study. Cardiovasc Diabetol 2007;6:13.

3 Grundy SM, Cleeman JI, Daniels SR, Donato KA, Eckel RH, Franklin BA, Gordon DJ, Krauss RM, Savage PJ, Smith SC Jr, Spertus JA, Costa F: Diagnosis and management of the metabolic syndrome: an American Heart Association/National Heart, Lung, and Blood Institute scientific statement. American Heart Association; National Heart, Lung, and Blood Institute. Circulation 2005;112:2735-2752.
4 Fachkommission Diabetes Sachsen, Saxony, Germany: Practical Guideline Metabolic Vascular Syndrome. 1st edition: 2007; 2nd edition: 2013.

5 Scholz GH: BOT-Basal(insulin) unterstützte orale Therapie bei Diabetes mellitus Typ 2 - Pathophysiologisch begründete Therapie bei Metabolisch-Vaskulärem Syndrom (MVS). Bremen, UNI-MED, 2010.

6 Hanefeld M, Pistrosch F, Bornstein SR, Birkenfeld AL: The metabolic vascular syndrome - guide to an individualized treatment. Rev Endocr Metab Disord 2016; 17:5-17.

7 Shin JA, Lee JH, Lim SY, Ha HS, Kwon HS, Park YM, Lee WC, Kang MI, Yim HW, Yoon KH, Son HY: Metabolic syndrome as a predictor of type 2 diabetes, and its clinical interpretations and usefulness. J Diabetes Investig 2013;4:334-343.
8 Alberti KG, Eckel RH, Grundy SM, Zimmet PZ, Cleeman JI, Donato KA, Fruchart JC, James WP, Loria CM, Smith SC Jr; International Diabetes Federation Task Force on Epidemiology and Prevention; National Heart, Lung, and Blood Institute; American Heart Association; World Heart Federation; International Atherosclerosis Society; International Association for the Study of Obesity: Harmonizing the metabolic syndrome: a joint interim statement of the International Diabetes Federation Task Force on Epidemiology and Prevention; National Heart, Lung, and Blood Institute American Heart Association; World Heart Federation; International Atherosclerosis Society; and International Association for the Study of Obesity. Circulation 2009;120:1640-1645. 
9 Reaven GM: Banting lecture 1988. Role of insulin resistance in human disease. Diabetes 1988;37:1595-1607.

10 DeFronzo RA, Ferrannini E: Insulin resistance. A multifaceted syndrome responsible for NIDDM, obesity, hypertension, dyslipidemia, and atherosclerotic cardi ovascular disease. Diabetes Care 1991;14:173-194.

11 Cefalu WT: Insulin resistance: cellular and clinical concepts. Exp Biol Med (Maywood) 2001;226:13-26.

12 Samuel VT, Shulman GI: The pathogenesis of insulin resistance: integrating signaling pathways and substrate flux. J Clin Invest 2016;126:12-22.

13 Brown MS, Goldstein JL: Selective versus total insulin resistance: a pathogenic paradox. Cell Metab 2008;7: 95-96.

14 Li S, Brown MS, Goldstein JL: Bifurcation of insulin signaling pathway in rat liver: mTORC1 required for stimulation of lipogenesis, but not inhibition of gluconeogenesis. Proc Natl Acad Sci U S A 2010;107:34413446.

15 Titchenell PM, Quinn WJ, Lu M, Chu Q, Lu W, Li C, Chen H, Monks BR, Chen J, Rabinowitz JD, Birnbaum MJ: Direct hepatocyte insulin signaling is required for lipogenesis but is dispensable for the suppression of glucose production. Cell Metab 2016;23:1154-1166.

D16 Tan SX, Fisher-Wellman KH, Fazakerley DJ, Ng Y, Pant H, Li J, Meoli CC, Coster AC, Stöckli J, James DE: Selective insulin resistance in adipocytes. J Biol Chem 2015;290:11337-11348.

17 Schmidt V, Schulz N, Yan X, Schürmann A, Kempa S, Kern M, Blüher M, Poy MN, Olivecrona G, Willnow TE: SORLA facilitates insulin receptor signaling in adipocytes and exacerbates obesity. J Clin Invest 2016; 126:2706-2720.

18 Kumari M, Wang X, Lantier L, Lyubetskaya A, Eguchi J, Kang S, Tenen D, Roh HC, Kong X, Kazak L, Ahmad $\mathrm{R}$, Rosen ED: IRF3 promotes adipose inflammation and insulin resistance and represses browning. J Clin Invest 2016;126:2839-2854.

19 Jiao P, Ma J, Feng B, Zhang H, Diehl JA, Chin YE, Yan $\mathrm{W}, \mathrm{Xu} \mathrm{H}$ : FFA-induced adipocyte inflammation and insulin resistance: involvement of ER stress and IKK $\beta$ pathways. Obesity (Silver Spring) 2011;19:483-491.

-20 Vergoni B, Cornejo PJ, Gilleron J, Dedjani M, Ceppo F, Jacquel A, Bouget G, Ginet C, Gonzalez T, Maillet J, Dhennin V, Verbanck M, Auberger P, Froguel P, Tanti JF, Cormont M: DNA damage and the activation of the p53 pathway mediate alterations in metabolic and secretory functions of adipocytes. Diabetes 2016;DOI: 10.2337/db16-0014.

21 King GL, Park K, Li Q: Selective insulin resistance and the development of cardiovascular diseases in diabetes: the 2015 Edwin Bierman Award Lecture. Diabetes 2016 65:1462-1471.

22 Cefalu WT: 'Prediabetes': are there problems with this label? No, we need heightened awareness of this condition! Diabetes Care 2016;39:1472-1477.

23 Yudkin JS: 'Prediabetes': are there problems with this label? Yes, the label creates further problems! Diabetes Care 2016;39:1468-1471.

24 Scherer PE: The multifaceted roles of adipose tissuetherapeutic targets for diabetes and beyond: the 2015 Banting Lecture. Diabetes 2016;65:1452-1461.

25 Sims EA: Are there persons who are obese, but metabolically healthy? Metabolism 2001;12:1499-1504

26 Blüher M: The distinction of metabolically 'healthy' from 'unhealthy' obese individuals. Curr Opin Lipidol 2010;1:38-43.

27 Muñoz-Garach A, Cornejo-Pareja I, Tinahones FJ: Does metabolically healthy obesity exist? Nutrients 2016;8:pii: E320.
28 Zheng R, Liu C, Wang C, Zhou B, Liu Y, Pan F, Zhang R, Zhu Y: Natural course of metabolically healthy overweight/obese subjects and the impact of weight change. Nutrients 2016;8:pii: E430.

29 Gonçalves CG, Glade MJ, Meguid MM: Metabolically healthy obese individuals: key protective factors. $\mathrm{Nu}$ trition 2016;32:14-20.

30 Rydén M, Hrydziuszko O, Mileti E, et al: The adipose transcriptional response to insulin is determined by obesity, not insulin sensitivity. Cell Rep 2016;16:23172326.

31 Lopes HF, Corrêa-Giannella ML, Consolim-Colombo FM, Egan BM: Visceral adiposity syndrome. Diabetol Metab Syndr 2016;8:40.

32 Ebrahimi-Mamaeghani M, Mohammadi S, Arefhosseini SR, Fallah P, Bazi Z: Adiponectin as a potential biomarker of vascular disease. Vasc Health Risk Manag 2015;11:55-70.

33 Jornayvaz FR, Shulman GI: Diacylglycerol activation of protein kinase $\mathrm{C} \varepsilon$ and hepatic insulin resistance. Cell Metab 2012;15:574-584.

34 Hotamisligil GS: Inflammation and metabolic disorders. Nature 2006;444:860-867.

35 Blüher M: Adipose tissue inflammation: a cause or consequence of obesity-related insulin resistance. Clinical Science 2016;130:1603-1614.

36 Moreno-Indias I, Tinahones FJ: Impaired adipose tissue expandability and lipogenic capacities as one of the main causes of metabolic disorders. J Diabetes Res 2015;2015:970375.

37 Blüher M: Adipose tissue dysfunction contributes to obesity-related metabolic diseases. Best Pract Res Clin Endocrinol Metab 2013;27:163-177.

38 Seven E, Husemoen LL, Sehested TS, Ibsen H, Wachtell K, Linneberg A, Jeppesen JL: Adipocytokines, Creactive protein, and cardiovascular disease: a population-based prospective study. PLoS One 2015;10: e0128987.

39 Wolk R, Bertolet M, Singh P, Brooks MM, Pratley RE, Frye RL, Mooradian AD, Rutter MK, Calvin AD, Chaitman BR, Somers VK; BARI 2D Study Group: Prognostic value of adipokines in predicting cardiovascular outcome: explaining the obesity paradox. Mayo Clin Proc 2016;91:858-866.

$40 \mathrm{Wu}$ ZJ, Cheng YJ, Gu WJ, Aung LH: Adiponectin is associated with increased mortality in patients with already established cardiovascular disease: a systematic review and meta-analysis. Metabolism 2014;63:11571166.

41 Ortega Moreno L, Copetti M, Fontana A, De Bonis C, Salvemini L, Trischitta V, Menzaghi C: Evidence of a causal relationship between high serum adiponectin levels and increased cardiovascular mortality rate in patients with type 2 diabetes. Cardiovasc Diabetol 2016;15:17.

42 Gotoda T: Another paradox regarding adiponectin revisited. J Atheroscler Thromb 2016;23:292-294.

43 Yang RZ, Lee MJ, Hu H, Pray J, Wu HB, Hansen BC, Shuldiner AR, Fried SK, McLenithan JC, Gong DW: Identification of omentin as a novel depot-specific adipokine in human adipose tissue: possible role in modulating insulin action. Am J Physiol Endocrinol Metab 2006;290:E1253-1261.

44 Greulich S, Chen WJ, Maxhera B, Rijzewijk LJ, van der Meer RW, Jonker JT, Mueller H, de Wiza DH, Floerke RR, Smiris K, Lamb HJ, de Roos A, Bax JJ, Romijn JA, Smit JW, Akhyari P, Lichtenberg A, Eckel J, Diamant M, Ouwens DM: Cardioprotective properties of omentin-1 in type 2 diabetes: evidence from clinical and in vitro studies. PLoS One 2013;8:e59697.
45 Tan YL, Zheng XL, Tang CK: The protective functions of omentin in cardiovascular diseases. Clin Chim Acta 2015;448:98-106.

46 Du Y, Ji Q, Cai L, Huang F, Lai Y, Liu Y, Yu J, Han B, Zhu E, Zhang J, Zhou Y, Wang Z, Zhao Y: Association between omentin-1 expression in human epicardial adipose tissue and coronary atherosclerosis. Cardiovasc Diabetol 2016;15:90.

47 Saely CH, Leiherer A, Muendlein A, Vonbank A, Rein $\mathrm{P}$, Geiger K, Malin C, Drexel H: Coronary patients with high plasma omentin are at a higher cardiovascular risk. Data Brief 2015;6:158-161.

48 Maffei M, Halaas J, Ravussin E, Pratley RE, Lee GH, Zhang Y, Fei H, Kim S, Lallone R, Ranganathan S, Kern PA, Friedman JM: Leptin levels in human and rodent: measurement of plasma leptin and ob RNA in obese and weight-reduced subjects. Nat Med 1995; 1: 1155-1161.

49 Scholz GH, Englaro P, Thiele I, Scholz M, Klusmann T, Kellner K, Rascher W, Blum WF: Dissociation of serum leptin concentration and body fat content during long term dietary intervention in obese individuals. Horm Metab Res 1996;28:718-723.

50 Friedman JM, Mantzoros CS: 20 years of leptin: from the discovery of the leptin gene to leptin in our therapeutic armamentarium. Metabolism 2015;64:1-4.

51 Xue B, Yu Y, Zhang Z, Guo F, Beltz TG, Thunhorst RL, Felder RB, Johnson AK: Leptin mediates high-fat diet sensitization of angiotensin II-elicited hypertension by upregulating the brain renin-angiotensin system and inflammation. Hypertension 2016;67:970-976.

52 Tsay YC, Chen CH, Pan WH: Ages at onset of 5 cardiometabolic diseases adjusting for nonsusceptibility: implications for the pathogenesis of metabolic syndrome. Am J Epidemiol 2016;184:366-377.

53 Schwartz SS, Epstein S, Corkey BE, Grant SF, Gavin JR 3rd, Aguilar RB: The time is right for a new classification system for diabetes: rationale and implications of the $\beta$-cell-centric classification schema. Diabetes Care 2016;39:179-186.

54 Xiao C, Dash S, Morgantini C, Hegele RA, Lewis GF: Pharmacological targeting of the atherogenic dyslipidemia complex: the next frontier in CVD prevention beyond lowering LDL cholesterol. Diabetes 2016;65: 1767-1778.

55 Ecelbarger CM: Metabolic syndrome, hypertension, and the frontier between. Am J Physiol Renal Physiol 2016;310:F1175-1177.

56 Frigolet ME, Torres N, Tovar AR: The renin-angiotensin system in adipose tissue and its metabolic consequences during obesity. J Nutr Biochem 2013;24: 2003-2015.

57 Ehrhart-Bornstein M, Lamounier-Zepter V, Schraven A, Langenbach J, Willenberg HS, Barthel A, Hauner H, McCann SM, Scherbaum WA, Bornstein SR: Human adipocytes secrete mineralocorticoid-releasing factors. Proc Natl Acad Sci U S A 2003;100:14211-14216.

58 Vleugels K, Schinner S, Krause D, Morawietz H, Bornstein SR, Ehrhart-Bornstein M, Krug AW: ERK1/2 MAPKs and Wnt signaling pathways are independently involved in adipocytokine-mediated aldosterone secretion. Exp Clin Endocrinol Diabetes 2011;119: 644-648.

59 O'Seaghdha CM1, Hwang SJ, Vasan RS, Larson MG, Hoffmann U, Wang TJ, Fox CS: Correlation of renin angiotensin and aldosterone system activity with subcutaneous and visceral adiposity: the Framingham Heart Study. BMC Endocr Disord 2012;12:3. 